\title{
Virtual Reality: A Tool for Managing and Mitigating the Nursing Fatigue
}

\section{Sohayb ABU TAPANJEH ${ }^{1}$, Mahmoud Al-Masaeed ${ }^{2}$ and Muhammad Alqudah ${ }^{3 *}$}

${ }^{1}$ Researcher, RN, MSN and PhD candidate, School of Nursing and Midwifery, Faculty of Health and Medicine, Address: University of Newcastle, Callaghan NSW 2308, Australia

${ }^{2}$ Researcher, RN and PhD Candidate, School of Nursing and Midwifery, College of Health, Medicine and Wellbeing, University of Newcastle, Callaghan, Newcastle 2308 Australia, and Faculty of Medicine and Health Sciences, Universiti Putra Malaysia, Serdang, Selangor, Malaysia ${ }^{3}$ Lecturer at the University of Newcastle - School of Nursing and Midwifery, Faculty of Health and Medicine, Australia

*Corresponding author: Mahmoud Al-Masaeed, Researcher, RN and PhD candidate, School of Nursing and Midwifery, Faculty of Health and Medicine, University of Newcastle, Callaghan NSW 2308 Australia, Tel: +61(0)470570566; Email: Mahmoud. almasaeed@uon.edu.au

\section{Abstract}

Background and Aim: Nurses' fatigue risks are on the rise. The implications include a rise in nurse's dissatisfaction, a decline in nurses' health, and a rise in the risk of medical errors. There is a need to counter fatigue by either coping with it or eliminating the risks involved. One of the emerging alternatives to handling nursing fatigue is virtual reality (VR) technology. The review examines the existing literature on the use of VR in managing nurses' fatigue.

Methods: The review adopts an ILR approach. This includes collecting relevant literature online and critically examining it for patterns and trends on the topic. The required literature is collected from four databases, CINAHL, Google Scholar, Scopus, and Medline. An electronic search strategy for the Scopus database is duplicated across the other three databases. The findings result in MEDLINE (16), Google Scholar (41), SCOPUS (33), and CINAHL (19). After screening through the PRISMA Equator tool and a GRADE model on the articles' quality and reliability, 21 articles were used in the study analysis.

Findings and Conclusion: The review findings indicate three broad areas of VR application in managing nurses' fatigue. They are (i) VR in encouraging nurses' rest, (ii) VR in promoting nurses' psychological well-being, and (iii) VR in nurses' fatigue mitigation training. The overall review conclusion is that VR is emerging as a complementary tool in managing nurses' fatigue. Institutions should invest in VR tools and infrastructure to complement their current nurses' fatigue mitigation and coping strategies.

Keywords: Virtual Reality; Nurse; Fatigue; Coping; Management

\section{Introduction}

Fatigue is a state of exhaustion and tiredness. When an employee is exhausted, they cannot function and execute their duties and responsibilities to the optimum [1,2]. When the nurses are exhausted, they cannot execute their professional and personal life responsibilities as expected. There exist different types of nurses' fatigue. They are categorised based on the occurrence period and the fatiguing nature. On the one hand, the Occupational fatigue exhaustion recovery 
tool (OFER-15) categories nurses fatigue into three distinct types, namely the acute (occurring within a shift), chronic fatigue (occurs over a more extended period), and intershift recovery fatigue (due to the lack of enough rest time between shifts) $[3,4]$. On the other hand, fatigue is described based on the nature of the impact. The fatigue assessment scale tool (FAS) categorises fatigue into two levels, the physical (affecting the physical functioning and health) and mental fatigue (impacting on the nurses' psychological wellbeing) $[5,6]$. Regardless of the type of fatigue experienced, fatigue lowers the nurses' output and productivity, directly impacting the patients' quality of Healthcare offered and experienced.

The nurses are the strategic and primary point of contact for the patients when seeking healthcare services, thus improving their optimal functioning. The implications of nurses fatigue range from personal (deteriorating health) through social (declining family and social relations) to professional (lack of concentration and increased risk of medical errors) [7]. The need to eliminate the implications of fatigue necessitates the development and adoption of fatigue mitigation strategies [5-9]. These are strategies aimed at eliminating fatigue risks among nurses or mitigating the fatigue levels to lower and manageable levels.

The strategies vary from an individual (those initiated by the nurses) to institutional (policy level strategies instituted by the institutions and respective governments and responsible bodies) [8-10]. The emergence of technology has improved healthcare delivery and, as such improved healthcare services quality. One among the areas it is applied is in include nurses training and management. One of its application's emerging areas is a management tool in handling and mitigating nurses' fatigue [11,12].

This review develops an efficacy analysis of the virtual reality (VR) technology as a nurse's fatigue management and coping strategy and tool. Virtual reality is a scientific simulation allowing individuals to interact with a virtually create an environment in a three-dimensional context. VR simulation has evolved from the traditional use of sight senses to the hearing and even feeling and touch feelings use [13]. This highlights the technology gains and value in addressing nurses' fatigue compared to earlier non-technology-based coping strategies. Further, it highlights weakness areas in the current VR usage as fatigue coping and management tool.

\section{Aims}

The study examines the efficacy and value of virtual reality as a nurses fatigue coping strategy. The review examines the VR application's success and existing and projected challenges in coping with nurse's fatigue.

\section{Materials and Methods}

The review adopted an integrated Literature review (ILR) approach. This was based on the need to examine and critically compare and contrast existing literature on the topic. The focus was on VR use in managing and coping with nurses' fatigue. As a relatively new nursing management concept, literature has been developed focusing on specific contexts and areas of nursing fatigue. The development of ILR reviews enables the critical comparison and contrasting of data, thus enabling refined and additional knowledge on the subject area $[14,15]$. In collecting and analysing the required review data, the Whittemore and Knafl, et al. framework was applied in this review's development. It comprises five steps, including (1) identifying the purpose of the review, (2) searching for the relevant articles, (3) evaluating and extracting data, (4) analysing or synthesising the data, and (5) presenting the findings. The literature search was developed through an electronic search. The review searched four databases, namely CINAHL, Medline, Scopus, and Google Scholar, respectively. The databases were selected based on their article's diversity and the presence of nurses and nursing fatigue-related articles. The ILR review period of review was within the last decade, thus focusing on literature published from 2010-2020. Further, any emerging studies published in the early months of 2021 were equally considered.

An electronic search was developed on the Scopus database and was then replicated across the other three databases [16]. The keywords and phrases used included 'nurs*” and 'fatigue' OR 'lethargy' OR 'tired' AND 'virtual reality' OR 'VR' AND 'coping.' The article search and collection process was based on inclusion criteria for only the review studies published after 2010 (maximum a decade ago). The publication time limitation ensured only relevant, and current articles were adopted for analysis. Additionally, the used articles were published in the English language or had an English language version and were available in full PDFs. Moreover, the study articles were excluded if they were published earlier than 2010 or lacked a full PDF file for analysis.

The obtained articles were analysed for quality and inclusion reliability in the review. The review applied the GRADE quality assessment model. The model assesses the quality of articles based on their relevance to the review aim and focus, the risk of researcher bias, and the article's findings' accuracy. The grading model ranks the articles on a scale ranging from high, moderate, low and/or very low $[17,18]$. For inclusion in the review study, articles should have a minimum, moderate ranking. This is to ensure that only accurate, focused on the review aim and without a researcher's bias are included in a review. The final step in 
the review was in data extraction and analysis. The PRISMA EQUATOR tool was used to ensure that all the steps in the review were adhered to, undertaken, and followed as required in developing an ILR review [19]. Additionally, the extraction process was thematically based on the key findings on the article's publication date, country of origin, methodologies, aims, key findings, and implications data extracted into an excel file before analysis and presentation in the review findings.

\section{Results}

After a comprehensive search of the articles, the search resulted in an initial MEDLINE (16), Google Scholar (41), SCOPUS (33), and CINAHL (19). After applying inclusion and exclusion criteria and quality appraisal, 21 articles were selected for inclusion in the review findings and analysis. Although the obtained findings had different thematic focus, the key emerging themes in relation to the review focus were (i) VR in encouraging nurses' rest, (ii) VR in promoting nurses' psychological well-being, and (iii) VR in nurses' fatigue mitigation training. PRISMA model for the findings is illustrated in the figure 1 below.

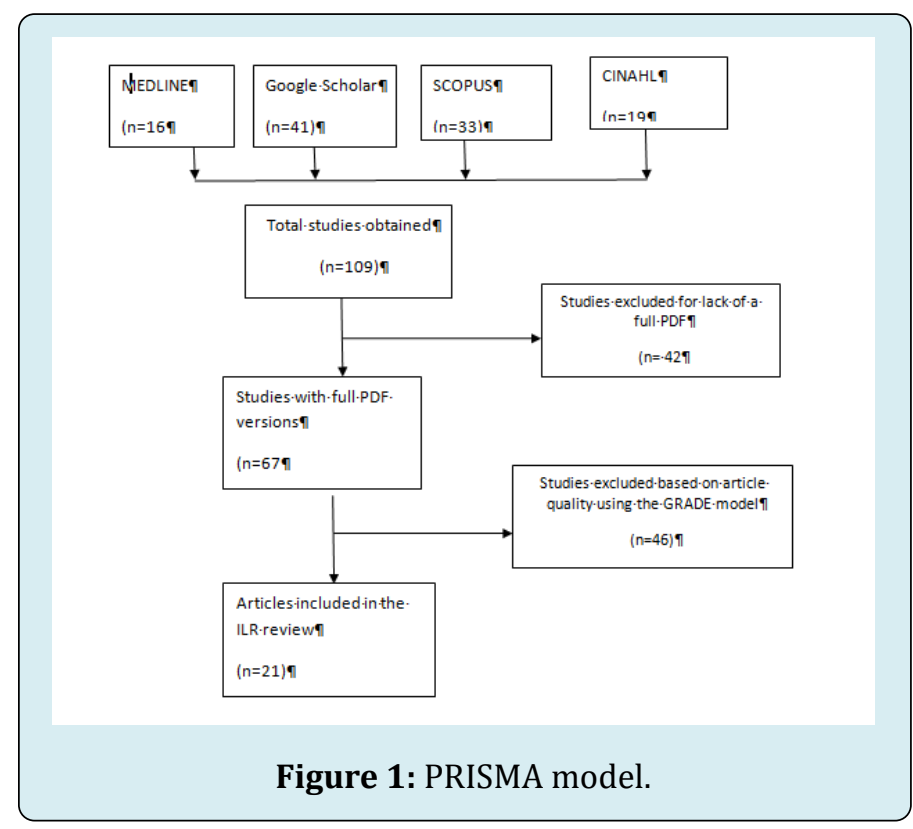

VR in encouraging Nurses Rest

The obtained findings indicated that VR was increasingly used as an entertainment tool. VR has an entertainment component to allow for the playing of interactive games $[11,12,20,21]$. Unlike the traditional games that were isolated and offline, VR allows and enables players to interact and engage in real-time game sessions. This has a lasting effect on the nurses' ability to relax. With the installation of VR applications and equipment in nurses' rest areas, the nurses have an opportunity to engage VR during their off shift and in between the shift rest periods $[11,12,22]$.

The nurses' shift structures allow for both off the shift resting periods and in-the-shift breaks. During the breaks, the nurses must rejuvenate and re-energize to meet their professional obligations during the shifts [11]. The installation of VR tools and devices for use during such breaks allows for, among other gains, mental relaxation. The nurses get their mind off the shift and allow for refreshment and relaxation during the breaks. Additionally, this is useful on the off the shift rest periods, as it offers an additional hobby for the nurses [22-24].

\section{VR in promoting Nurses Psychological Wellbeing}

Studies demonstrated a link between nurses' use of VR devices and tools and improved psychological wellbeing. Studies reporting the findings were mainly focused on the nurses' mental fatigue and their psychological state $[22,24,25]$. VR serves as an escape route for the nurses' from their current state of tiredness and fatigue. In most instances, the studies demonstrated that their professional responsibilities overburdened the nurses and lacked enough time for their personal life and social experiences. The lack of social interactions has an impact on the nurses' mental well-being, leading to the lack of concentration and the risk of medical errors [26,27]. These challenges are partially addressed through the use of VR applications. VR allows for the use of fully immersed experiences.

The full immersion simulation of real-life social experience offers the nurse san opportunity to experience social adventures such as enjoying holidays and other simulated situations. In the long run, the application offers an opportunity for the nurses to experience the gains of the other traditional coping strategies such as vacationing and holidaying through the use of VR [28-30]. An instance is the use of VR simulations on global and world-renowned tourists' sites and destinations. The VR platform offers the nurses an opportunity to virtually experience the destinations while addressing and meeting their professional responsibilities $[23,30,31]$. The elimination of transport and logistical needs and the time to travel to such destinations improve the nurses' work-life balance.

\section{VR on Nurses Fatigue Mitigation Training}

Articles indicated the increasing use of VR as a nurse training tool. The findings demonstrated that VR has emerged as a critical component in training nurses and increasing their quality of care $[24,29,32]$. The use of VR training has promoted the use of an evidence-based approach in training the nurses. Instances included in training mental healthcare 
nurses and training nurses on efficient task execution. For mental health nurses, the use of VR improves their preparedness to handling and dealing with mental health patients. Thus, they are prepared and have a simulated experience in handling their erratic behaviour and managing their conditions [26,33]. This helps in mitigating the risk and rise in their mental fatigue. Equally, the application extends to the critical care and intensive care unit nurses and the emergency department nurses. The use of VR in training prepares them for emergencies and patients facing lifethreatening conditions. This has an equal effect in managing and lowering their mental health risk [32].

Additionally, studies indicated that VR training improved nurse efficiency; by allowing them to practice their task execution. Increased practice and trials enable them to improve on-task execution efficiency. In the long run, this lowers the requirements of their physical tasks and energy level and, consequently, lowers fatigue levels during and after shifts $[12,26]$. Further, the findings demonstrated that VR training improved the nurses' rest by training and equipping them with the right set of skills on relaxation and managing their off the limited shift time.

\section{Discussion}

A critical evaluation of the ILR review findings indicates the existence of a relationship between nursing fatigue management and the use of VR. The findings indicate that the use of VR helps in addressing the different types of nurses' fatigue. On the one hand, VR as a rest support tool helps the nurses managing their physical and mental fatigue [24,34]. On the one hand, mental fatigue, often manifested in the decline and loss of concentration and apathy, emerges due to repeated execution of a set of responsibilities with minimal breaks. The use of VR devices and tools in games offers a distraction for the nurses' from their highly engaging patient needs $[4,35]$.

The simulated environment creates a space where the nurses can exist without the concerns and worries of their patients' demands and needs. Consequently, this helps in easing and relieving off their mental and psychological pressure. The effect is a rejuvenated mind and as such, a reduction and mitigation of the nurses' mental fatigue risks. Moreover, with VR simulation for virtual tours and experiences such as visiting key global destinations, having social interactions, and vacationing, the nurses' mental wellbeing is significantly improved. This has the long-term effect of creating an enhanced nurses' work-life balance [36,37].

One of the triggers of nurses' mental and physical fatigue is the lack of a work-life balance due to the workplace's professional demands; the nurses rarely have enough social- life time. Consequently, they are often overburdened by their career responsibilities and end up giving up their social life endeavours such as vacationing and visiting leading destinations [36,37]. The use of VR enables creating a balance by allowing both availability at the workplace and a social life boast through virtual and simulated engagements. Equally, the use of the VR games and fully immersive simulation helps in creating physical relief. An engagement in games and simulated social experiences helps the nurses ease the work pressure by serving as a rest period. In the long run, this plays a crucial role in easing the nurses' physical fatigue in reduced chronic and acute fatigue types [38,39].

Additionally, the findings demonstrate the value of VR as fatigue management and coping strategy tool. First, the use of VR training lowers the nurses' mental fatigue levels. The VR process offers the nurses training an opportunity through a simulated experience on the interaction with the patients. This serves as psychological preparedness tool for the nurses before they engage with the clients. Since the nurses already understand the nature and context of the patients' needs and demands, the risk of mental fatigue and psychological disturbances is significantly reduced. Secondly, the use of VR in training helps in equipping the nurses with practice efficiency. In most instances, studies demonstrate that chronic and acute fatigue types that are a manifestation of physical fatigue emerge not only due to the workload that the nurses have to handle but due to how they handle the responsibilities [26,29].

A lack of experience in tasks and duties increase the risks of fatigue. This explains why experienced nurses often register a lower acute fatigue index than the less experienced nurses. The use of VR training serves to prove the required nurses' experience through simulated environments. Thus, it eliminates the experience gap and enables even the less experienced nurses' efficiency in handling tasks, thus reducing fatigue [40]. Finally, VR training offers an essential fatigue mitigation and coping strategies insight. Studies demonstrated that poor rest management skills were among the leading causative factors $[24,40,41]$. The use of VR helps in addressing the challenge. Through simulated training, nurses are equipped with critical skills such as improving quality of sleep and the need and rationale for exercising. The net effect is an effective off-the-shift management strategy, thus lowering the nurses' inter-shift recovery fatigue levels.

\section{Practice Application and Limitations}

An evaluation of the study findings demonstrates the relevance of VR as a nurse's fatigue mitigation strategy and tool. The findings show that VR applications can be adopted as entertainment, a virtual interaction, and a training tool. The different applications help in addressing and mitigating 
different types of nurses' fatigue. Thus, in its application, a clinical and Management decision is critical to ensure that the application form and context meets the target nurses' fatigue type.

Despite the review contributions to the nursing fatigue management and coping literature, it has its share of limitations. One of the critical limitations in the review was its literature diversity on regional focus. The review lacked a specific regional focus and instead analysed the literature in a general global context. However, although fatigue remains a global nursing management concern, existing studies indicate a variance in the levels and intensity of nurses' fatigue across nations and regions. Future reviews and studies in relation to the review findings should be based on specific regions examination. Secondly, the developed review was cross-sectional. It focused on the impacts of VR on nurses' fatigue levels. Future longitudinal studies should be developed after the review findings. They should focus on examining how the use of VR in the three identified states impacts nurses' fatigue.

\section{Conclusion}

In summary, the review has demonstrated the link between VR use and nurses' fatigue mitigation. VR as an entertainment tool during the shifts and the shift breaks helps re-energize them both mentally and physically. Equally, VR as a virtual simulation and interaction tool allows for mental well-being through promoting and enhancing a worklife balance. The virtual interactions the nurses have bridged their real-life work-life imbalances caused by increased workrelated demands. Finally, it demonstrates that VR training helps address the nurses' lack of awareness and knowledge on fatigue coping techniques. The review findings are a demonstration of the value of VR in nursing management. Unfortunately, the use of VR as a nursing management tool remains an underutilised resource. The reviews serve as a first step towards encouraging institutions and nurse managers to invest in VR as an additional and supportive tool in managing nurse fatigue into the future.

\section{Conflict of Interest}

There is no conflict of interest.

\section{Funding}

The review was not funded

\section{References}

1. Barker LM, Nussbaum MA (2011) Fatigue, performance and the work environment: a survey of registered nurses. Journal of advanced nursing 67(6): 1370-1382.

2. Smith Miller CA, Shaw Kokot J, Curro B, Jones CB (2014) An integrative review: fatigue among nurses in acute care settings. JONA: The Journal of Nursing Administration 44(9): 487-494.

3. Geiger Brown J, Rogers VE, Trinkoff AM, Kane RL, Bausell RB, et al. (2012) Sleep, sleepiness, fatigue, and performance of 12-hour-shift nurses. Chronobiology international 29(2): 211-219.

4. Hazzard B, Johnson K, Dordunoo D, Klein T, Russell B, et al. (2013) Work-and nonwork-related factors associated with PACU nurses' fatigue. Journal of PeriAnesthesia Nursing 28(4): 201-209.

5. Cella M, Chalder T (2010) Measuring fatigue in clinical and community settings. Journal of psychosomatic research 69(1): 17-22.

6. Roelen CA, Bültmann U, Groothoff J, Van Rhenen W, Magerøy N, et al. (2013) Physical and mental fatigue as predictors of sickness absence among Norwegian nurses. Research in nursing \& health 36(5): 453-465.

7. Al Masaeed M, Al Soud M, Alkhlaifat E, Alsababha R (2020) An Investigation of the Impacts of Covid-19 Pandemic Spread on Nurses Fatigue: An Integrated.

8. Pasupathy KS, Barker LM (2012) Impact of fatigue on performance in registered nurses: Data mining and implications for practice. Journal for Healthcare Quality 34(5): 22-30.

9. Steege LM, Pinekenstein BJ, Arsenault Knudsen É, Rainbow JG (2017) Exploring nurse leader fatigue: a mixed methods study. Journal of nursing management 25(4): 276-286.

10. Al Masaeed MAM, Al Motiry A, Alsababha RWM, Alqudah M, Ismail K (2020) Investigating nursing staff experiences and experts recommendations evolution as Covid-19 infection curve flattens globally: an integrated literature Review. IOSR Journal of Nursing and Health Science (IOSR-JNHS) pp: 2320-1959

11. Ferguson C, Davidson PM, Scott PJ, Jackson D, Hickman LD (2015) Augmented reality, virtual reality and gaming: an integral part of nursing. Contemp Nurse 51(1): 1-4.

12. Finley BA, Sheppard KG (2017) Compassion Fatigue: Exploring early-career oncology nurses' experiences. Clinical journal of oncology nursing 21(3): E61-E66.

13. Anthes C, Garcia Hernandez RJ, Wiedemann M, 
Kranzlmüller D (2016) State of the art of virtual reality technology. In 2016 IEEE Aerospace Conference pp: 1-19.

14. Van der Riet P, Levett Jones T, Aquino Russell C (2018) The effectiveness of mindfulness meditation for nurses and nursing students: An integrated literature review. Nurse education today 65: 201-211.

15. Oliveira SM, dD Alcantara Sousa LV, Gadelha MDSV, Do Nascimento VB (2019) Prevention actions of burnout syndrome in nurses: an integrating literature review. Clinical practice and epidemiology in mental health: CP \& EMH, 15: 64.

16. Chen L, Babar MA, Zhang H (2010) Towards an evidencebased understanding of electronic data sources. In 14th International Conference on Evaluation and Assessment in Software Engineering (EASE) pp: 1-4.

17. Armijo Olivo S, Stiles CR, Hagen NA, Biondo PD, Cummings GG (2012) Assessment of study quality for systematic reviews: a comparison of the Cochrane Collaboration Risk of Bias Tool and the Effective Public Health Practice Project Quality Assessment Tool: methodological research. Journal of evaluation in clinical practice 18(1): 12-18.

18. Paré G, Trudel MC, Jaana M, Kitsiou S (2015) Synthesizing information systems knowledge: A typology of literature reviews. Information \& Management 52(2): 183-199.

19. Tricco AC, Lillie E, Zarin $\mathrm{W}$, O'Brien $\mathrm{KK}$, Colquhoun $\mathrm{H}$, et al. (2018) PRISMA extension for scoping reviews (PRISMA-ScR): checklist and explanation. Annals of internal medicine 169(7): 467-473.

20. Lorenz VR, Sabino MO, Corrêa Filho HR (2018) Professional exhaustion, quality and intentions among family health nurses. Revista brasileira de enfermagem 71: 2295-2301.

21. Ioannou A, Papastavrou E, Avraamides MN, Charalambous A (2020) Virtual reality and symptoms management of anxiety, depression, fatigue, and pain: a systematic review. SAGE Open Nursing 6: 2377960820936163.

22. Brigham TJ (2017) Reality check: basics of augmented, virtual, and mixed reality. Medical reference services quarterly. 36(2): 171-178.

23. Triberti S, Repetto C, Riva G (2014) sychological factors influencing the effectiveness of virtual reality-based analgesia: a systematic review. Cyberpsychology, Behavior, and Social Networking, 17(6): 335-345.

24. Bracq MS, Michinov E, Jannin P (2019) Virtual reality simulation in nontechnical skills training for healthcare professionals: a systematic review. Simulation in Healthcare 14(3): 188-194.

25. Shah LBI, Torres S, Kannusamy P, Chng CML He, Klainin-Yobas P (2015) Efficacy of the virtual realitybased stress management program on stress-related variables in people with mood disorders: the feasibility study. Archives of psychiatric nursing, 29(1): 6-13.

26. Elliman J, Loizou M, Loizides F (2016) Virtual reality simulation training for student nurse education. In 2016 8th International Conference on Games and Virtual Worlds for Serious Applications (VS-Games) pp: 1-2.

27. Kidd LI, Knisley SJ, Morgan KI (2012) Effectiveness of a Second Life ${ }^{\circledR}$ simulation as a teaching strategy for undergraduate mental health nursing students. Journal of psychosocial nursing and mental health services, 50(7): 28-37.

28. Jenson CE, Forsyth DM (2012) Virtual reality simulation: using three-dimensional technology to teach nursing students. CIN: Computers, Informatics, Nursing 30(6): 312-318.

29. Farra SL, Smith S, Gillespie GL, Nicely S, Ulrich DL, et al. (2015) Decontamination training: With and without virtual reality simulation. Advanced emergency nursing journal 37(2): 125-133.

30. Dreesmann N (2018) Virtual reality check: Are you ready?. Journal of gerontological nursing 44(3): 3-4.

31. Lee H, Jung TH, Tom Dieck MC, Chung N (2020) Experiencing immersive virtual reality in museums. Information \& management 57(5): 103229.

32. Kilmon CA, Brown L, Ghosh S, Mikitiuk A (2010) Immersive virtual reality simulations in nursing education. Nursing education perspectives 31(5): 314317.

33. Dang BK, Palicte JS, Valdez A, O'Leary Kelley C (2018) Assessing simulation, virtual reality, and television modalities in clinical training. Clinical Simulation in Nursing 19: 30-37.

34. Pourmand A, Davis S, Lee D, Barber S, Sikka N (2017) Emerging utility of virtual reality as a multidisciplinary tool in clinical medicine. Games for Health Journal 6(5): 263-270.

35. Nuamah JK, Mehta RK (2020) Design for stress, fatigue, and workload management. In Design for health. Academic Press pp: 201-226. 
36. Khallash S, Kruse M (2012) The future of work and worklife balance 2025. Futures 44(7): 678-686.

37. Srivastava S, Kanpur R (2014) A study on quality of work life: key elements \& It's Implications. IOSR Journal of Business and Management 16(3): 54-59.

38. Perry B, Toffner G, Merrick T, Dalton J (2011) An exploration of the experience of compassion fatigue in clinical oncology nurses. Canadian Oncology Nursing Journal/Revue canadienne de soins infirmiers en oncologie 21(2): 91-97.
39. Barker LM, Nussbaum MA (2011) The effects of fatigue on performance in simulated nursing work. Ergonomics 54(9): 815-829.

40. Sitterding MC, Raab DL, Saupe JL, Israel KJ (2019) Using artificial intelligence and gaming to improve new nurse transition. Nurse Leader 17(2): 125-130.

41. Aebersold M, Tschannen D (2013) Simulation in nursing practice: The impact on patient care. The Online Journal of Issues in Nursing 18(2): 6. 